\title{
Research on the Method of Automatic Assembly for Load-Equipment of Aircraft Static Strength Test based on CAA
}

\author{
Xingsheng $\operatorname{Lin}^{1, a *}$, Shijun Zhou ${ }^{2, b}$ \\ ${ }^{1}$ Shenyang Aircraft Industries(Group) Co.,Ltd.,Shenyang, Liaoning, 110850, China. \\ ${ }^{2}$ Chinese people's Liberation Army in Shenyang aircraft industries (Group) Co., Ltd. Military \\ Representative Office, Shenyang, Liaoning, 110850, China \\ a 804922745@qq.com, bsj-zhou@sina.com
}

\begin{abstract}
Keywords: Automatic assembly; CAA; Strength Test; Positioning; CATIA
Abstract: Aiming at the problem that the installation of static strength test setup is not intuitive and it is difficult to check the existence of interference, this paper presents a method for automatic assembly of test loading equipment. The method is based on the CATIA two development technology CAA, the target coordinate system is created by the additional data generated by the test design, and then the precise positioning of the device is applied to realize the automatic assembly of the loading equipment. In this paper, the automatic assembly of the whole machine static strength test is verified. The results show that the method is feasible and effective.
\end{abstract}

\section{Introduction}

In the design phase of the static strength test of the aircraft, the installation diagram design is one of the important links. Due to the use of two-dimensional design method, the results of the design is not intuitive and difficult to check the presence of interference. The traditional solution is to find the problem in the place, the site modification, this approach may also lead to a small problem in order to solve a small problem, to modify a number of equipment installation, a serious impact on the progress of the test. At the same time, the test scene needs to load a lot of equipment, the efficiency is very low. At the same time, the test scene needs to load a lot of equipment, the efficiency is very low. For a device like a lever, it is not possible to rely on manual assembly, and the accuracy is not high.

Digital virtual assembly provides an effective way for the design of the installation drawing.The 3D digital model of virtual assembly can be used to design and test the test scenarios. The literature [1-3] using CATIA assembly module, the coaxial collinear and coplanar constraints, so as to realize the automatic assembly, the method in determining the location and the relationship between the reference element is relatively complicated. In this paper, a new method of automatic assembly of test loading equipment is proposed based on CATIA software CAA two. The first is based on the data of the lever installation diagram, extracting the spatial data of each equipment to form the target coordinate system, and then using the coordinate system transformation to achieve the precise positioning of each device, and then complete the automatic assembly of test loading equipment.

\section{The CAA development platform}

Component Application Architecture (CAA) component application architecture technology, which is a powerful tool for Systemes Dassault product development and customer development two. At present, Systemes Dassault has formed the six pillars of products, through the integration of HUB PPR, the life cycle of the product for a full range of management. Under the support of the CAA architecture, the Systemes Dassault system can be built as a building block, which is very suitable for the growth and development of the system. However, due to the complexity of the system and the deep content of CAA, users must have the knowledge and ability of the software development in the same time as the Systemes Dassault application system ${ }^{[4]}$. In order to realize the automatic assembly of the static strength test, in this paper, the framework of Document, Mathematics, PPR (Product/Process/Resources) is mainly used in the CAA framework, and the relationship between 
the product instance and the product reference is shown in Figure 1.

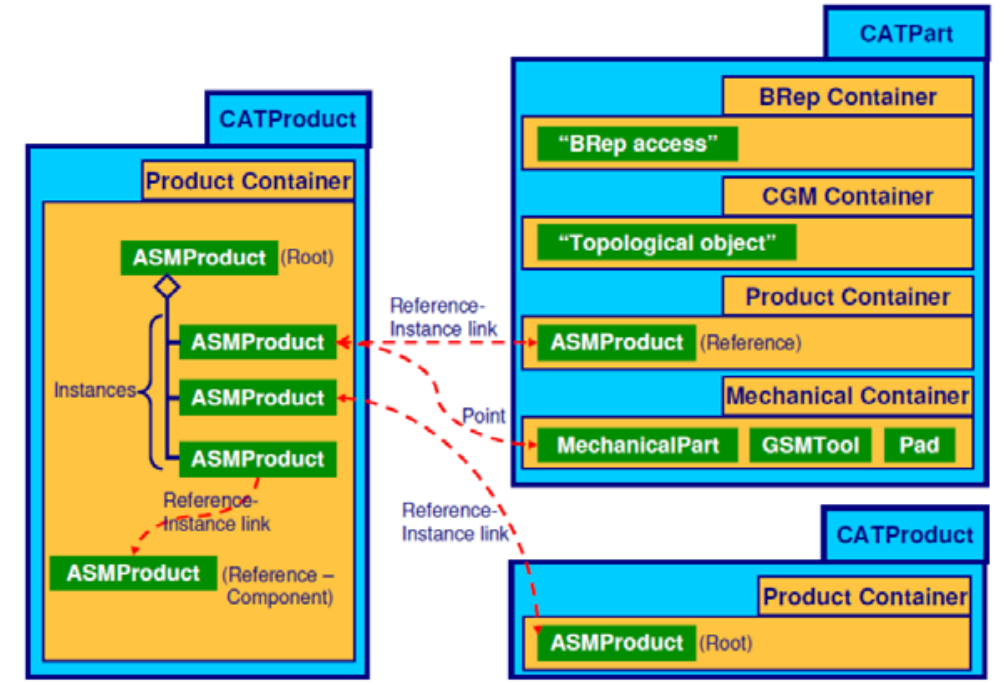

Figure 1.The relationship between the product instance and the product reference PPR

\section{Assembly positioning theory}

Local coordinate systems may vary from person to person when modeling the loading device. In this paper, the modeling of the test loading equipment is agreed before the assembly location is realized,such as a lever in the local coordinate system, the provisions on bolted joints of short arm as the origin of coordinates, the edge is $\mathrm{X}$ direction, $\mathrm{Y}$ direction direction of bolt hole. The connections in the local coordinate system, the provisions at the lower end of round center as the origin of coordinates, the length is $\mathrm{Z}$ direction, $\mathrm{Y}$ direction to guichenoti.

The principle for the construction of the target coordinate system is: each lever is connected with the next level to form a plane, that is, by combining the coordinates of the coordinates with the two connecting parts to form a three point plane, so that each lever has a plane corresponding to the three devices in the plane is not the same as the origin of the same target coordinates. With the above ideas, we can quickly build the target coordinate system for each equipment, and finally complete the assembly location.

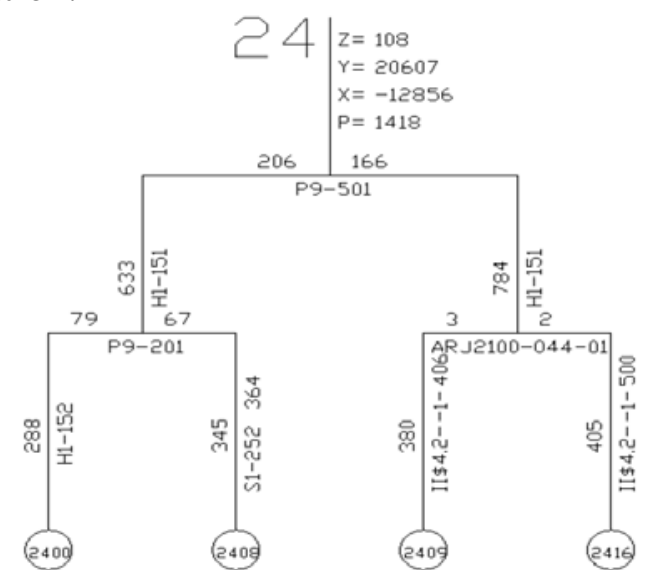

Figure 2.The equipment installation diagram

\section{Programming}

In order to realize the automatic assembly of the equipment, three key steps are involved in the process of the realization of the program: The first step is to use the programming language development assistance program to achieve data conversion, which is the original test design binary files (*.DAT) into the formatted data text file.The second step is to use CATIA two rapid development environment (Rapid, Application Development Environment RADE) and CAA 
language development with parameters of the implementation of the program, to achieve the load point for the unit, for each loading point of the test loading device used to call the CATIA assembly module for import, and in order to ensure the uniqueness of the device part number, the equipment is re number in the process of introduction; The third step is based on the first step to complete the design of the test data as the second step of the implementation of the parameters of the program to call,using Mathematics framework to complete the establishment of the target coordinate system, and according to the different equipment coordinate system transformation and positioning work ${ }^{[5-6]}$, and finally form the CATIA assembly file (*.CATProduct), the specific process is shown in Figure 3 .

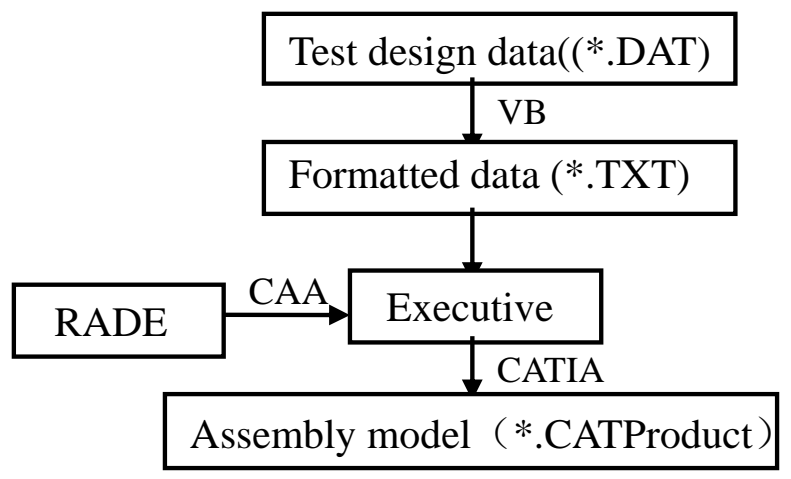

Figure3.Assembly document flow chart

The key procedures are as follows:

HRESULT rc = CATDocumentServices::OpenDocument(sFileToOpen, pPartDoc);

//Open parts documentation to be assembled.

SpProduct $=$ piProductOnRoot $->$ AddProduct(spRootPart);

//Use the CATIProduct interface pointer to add part documentation

CATIProduct_var spRef=piProductOnRoot-> GetReferenceProduct();

CATIProduct_var spProIns1=piInstanceProd1-> FindInstance(spRef);

// returns an instance of assembled parts in the product

CATIMovable * piMoveOnIns1=NULL;

rc=spProIns1->QueryInterface(IID_CATIMovable,(void**)\&piMoveOnIns1); ～//Transform

interface by instance access

CATMathAxis cAxis(CO,V3,V2,V1);// Construction target coordinate system

CATMathTransformation axisTrans = CATMathTransformation( CATMathOIJK, cAxis );//

Set up the transformation of coordinate system

rc=piMoveOnIns1->SetPosition(axisTrans,NULL_var);// Application transformation class to complete the localization

\section{Application examples}

Using CAA rapid development environment RADE to carry on the program development, and in the whole machine environment under a condition of the assembly as an example of automatic assembly, the working conditions with 66 points, involving the use of the lever, connecting pieces of thousands of, the assembly effect is shown in Figure 4. 


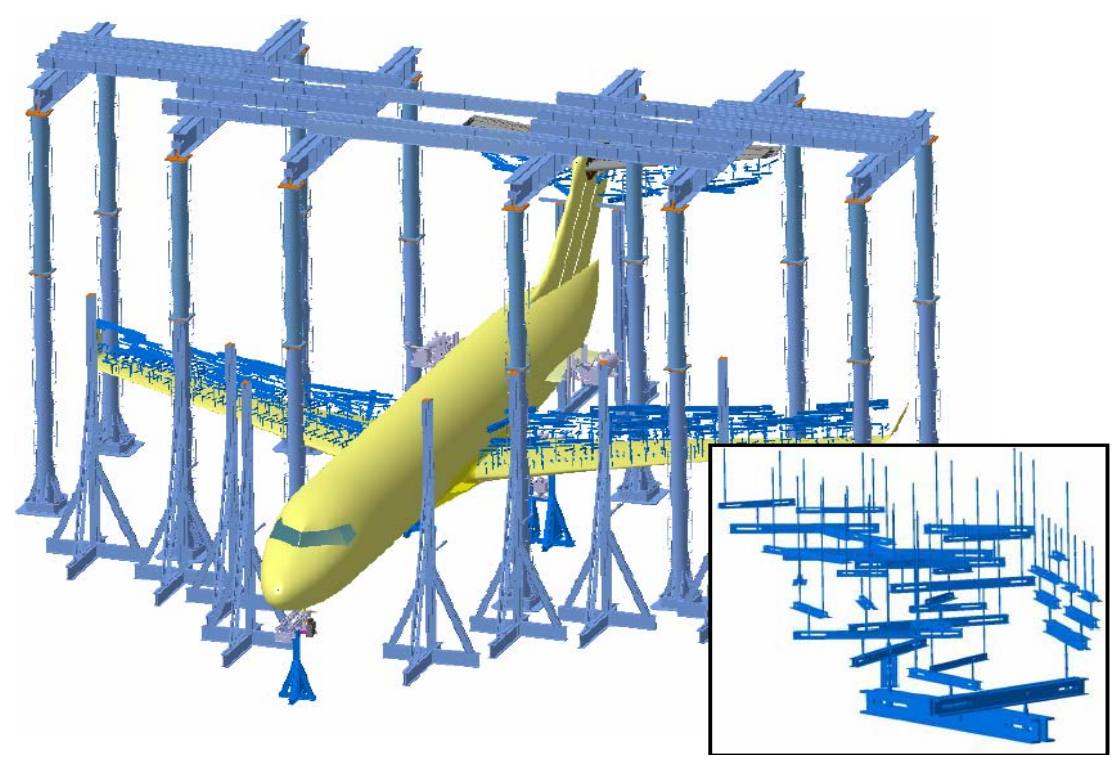

Figure 4. Assembly effect diagram

\section{Conclusions}

In this paper, through the research of CATIA two development CAA technology, this paper puts forward a method of automatic assembly of test loading equipment. Through the above examples and the actual application shows that the CAA technology can be used to realize the automatic assembly of test equipment. The method successfully solves the problem that the traditional two-dimensional design is not intuitive and difficult to identify the interference phenomenon. At the same time, compared with the traditional methods of using CATIA assembly module assembly, the method of this paper is relatively intuitive, it is easier to program implementation, and it has a high reference value for the development and application of the follow-up program.

\section{Reference}

[1] DING Zhiqiang ,ZHAO Cenxi, Automatic assembly of CATIA parameterized and intelligent technology based on[J]. Manufacturing Automation, 2005(8):52-53.

[2] LI Weixue, WANG Zhongqi, KANG Yonggang,etc, Automatic assembly of products based on V5 CATIA two[J]. Machinery Manufacturing, 2005(8):52-53.

[3] CHEN Guohua,CHENG Bensong,YANG Min, Virtual assembly method of mechanical parts based on CATIA[J]. Machine research and Application,2006,19(1):113-114.

[4] HE Chaoliang, DU Yanna, ZHANG Chao, Research on CATIA two development based on CAA[J]. Automation technology and Application 2006,25(9):37-39.

[5] Dassault Systemes. CAA V5 Encyclopedia Documents[EB/OL]. 2006.

[6] Dassault Systemes. CAA V5 For CATIA Getting Started[EB/OL]. 2005 